\title{
Pattern Recognition of Abnormal Left Ventricle Wall Motion in Cardiac MR
}

\author{
Yingli Lu ${ }^{1}$, Perry Radau ${ }^{1}$, Kim Connelly $^{1,2}$, Alexander Dick ${ }^{3}$, and Graham Wright ${ }^{1}$ \\ ${ }^{1}$ Imaging Research, Sunnybrook Health Sciences Centre, Toronto, ON, Canada \\ ${ }^{2}$ Cardiology, St Michael's Hospital, Toronto, ON, Canada \\ ${ }^{3}$ Cardiology, Sunnybrook Health Sciences Centre, Toronto, ON, Canada \\ yinglilu@gmail.com, perry.radau@gmail.com, connellyka@yahoo.ca, \\ Alexander.Dick@sunnybrook.ca, \\ gawrightasten. sunnybrook. utoronto.ca
}

\begin{abstract}
There are four main problems that limit application of pattern recognition techniques for recognition of abnormal cardiac left ventricle (LV) wall motion: 1) Normalization of the LV's size, shape, intensity level and position; 2) defining a spatial correspondence between phases and subjects; 3) extracting features; 4) and discriminating abnormal from normal wall motion. Solving these four problems is required for application of pattern recognition techniques to classify the normal and abnormal LV wall motion. In this work, we introduce a normalization scheme to solve the first and second problems. With this scheme, LVs are normalized to the same position, size, and intensity level. Using the normalized images, we proposed an intra-segment classification criterion based on a correlation measure to solve the third and fourth problems. Application of the method to recognition of abnormal cardiac MR LV wall motion showed promising results.
\end{abstract}

\section{Introduction}

Currently, diagnosis of abnormal left ventricle (LV) wall motion is generally based on visual inspection of the $4 \mathrm{D}(3 \mathrm{D} \times$ temporal $)$ dynamic cardiac magnetic resonance cine images. Studies have shown that this method may be inaccurate, time consuming and suffer from high inter-observer variability [1]. Therefore, methods of computer aided recognition of abnormal LV wall motion will clinically significant. Earlier literature $[1,2,3,4]$ on recognition of abnormal LV wall motion are generally based on enddiastolic (ED) and end-systolic (ES) phases' wall thickening and motion information. Goals of this work are to introduce a novel application of pattern recognition techniques for computer aided recognition of abnormal LV wall motion. Unlike previous approaches, it utilizes the wall thickness and motion information from all cardiac phases.

There are four main problems that limit application of pattern recognition techniques to the problem of detecting abnormal LV wall motion. First, the LV's size, shape, intensity level and position require normalization. A subject's LV changes size, shape, intensity level and position throughout the cardiac cycle. And there is additional 
inter-subject variation. Second, it is important to determine a spatial correspondence between phases and subjects. The LV wall motion features can be extracted only after defining this correspondence. The third problem is feature extraction that is sensitive to wall motion but not sensitive to the following factors: thickness variation of the myocardium across subjects, as well as the size and position variation of the papillary muscles and endocardial trabeculations across phases and subjects. The final problem is discrimination of normal and abnormal wall motion and selection of a classification criterion. Without solving these four problems, it is difficult to use pattern recognition techniques to detect and classify LV wall motion.

In this work, we introduce a normalization scheme so that the LV from each slice is normalized to polar coordinates with a fixed size, intensity level and position. Because cardiac motion is a complex combination of wall thickening, circumferential shortening and longitudinal ventricular shortening, determining pixel-based spatial registration phases and subjects is difficult and complicated by the requirement of retaining information about abnormal wall motion. This problem is simplified by establishing segment correspondence instead, and this appropriately matches clinical wall motion scoring practice[5,6]. In the feature extraction stage, we propose an intrasegment correlation to measure each segment's wall motion relative to normal values. Training data is used to determine the correlation measure criterion for discrimination of abnormal wall motion.

\section{Left Ventricle Normalization}

There are four steps in the normalization scheme: reference points localization, spatial normalization, intensity normalization, and labeling of segments. It is assumed that epicardial contours are available, for example after application of the automated segmentation algorithm described in the previous works [9-13].

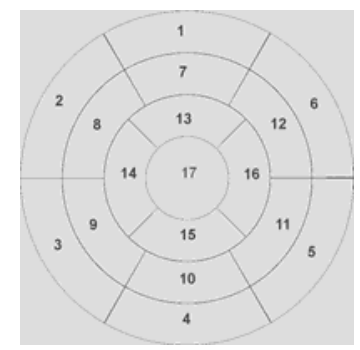

Fig. 1. AHA 17 myocardial segments

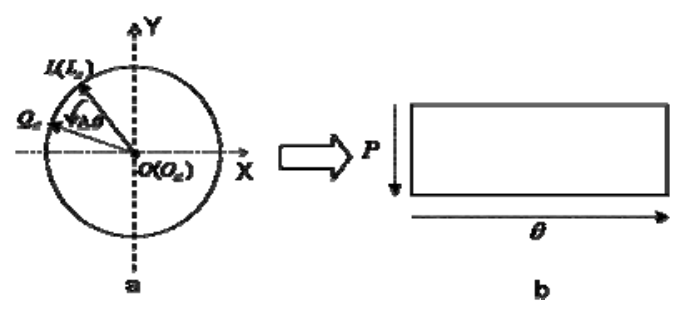

Fig. 2. Diagram of spatial normalization

Reference Points Localization. Because of the diversity of patients' body positions in the scanner and different heart positions in the thorax, we have to normalize different heart positions to the same. In this work, we used the AHA 17 segment reference $[5,6]$ (Refer to Fig.1). In which, the heart is divided into four parts cutting across the long axis of the LV: basal, mid-cavity, apical and apex. The basal and mid-cavity short axis slices are each divided radially into 6 segments, whereas the apical slice has 4 segments, and the 17th segment is the apex. According to the AHA 17 segment 
model, either junction between the right ventricular wall and the interventricular septum of the basal (and mid-cavity) slice on the ED phase can be used as reference point to identify different segments. In this work, the anterior end of the interventricular septum is used as the reference point. (Point $L$ in Fig. 3a and 3e). For each subject, there are two sub-steps to locate the reference points of each phase:

Step1. Mark reference point $L$ at the selected basal (or midcavity) slice's ED phase.

Step2. Calculate reference point of each phase (excluding ED). Given $L$ and centroid $O$ of the epicardium contour, we have the angle to the reference point $\mathrm{L}$ on the epicardium, $\angle X O L$ (Fig. 2a). For a given slice slice ${ }_{s t}$ and $\angle X O L$ and $O_{s t}$, we can get the reference point $L_{s t}$ of slice $_{s t}$ with the assumption that $\angle X O_{s t} L_{s t}=\angle X O L$ (Note: The slice at level $s$ and phase $t$ is defined as slice $e_{s t}$ $s=1,2 \ldots, S, t=1,2, \ldots, T$, where $S$ and $T$ are the maximum slice and phase numbers respectively. $O_{s t}$ is the centroid of the plotted epicardial contour of slice $\left._{\text {st }}\right)$.

Given reference points $L_{s t}$ of each subject, and combined with the "spatial normalization' step, heart positions of different subjects can be localized.
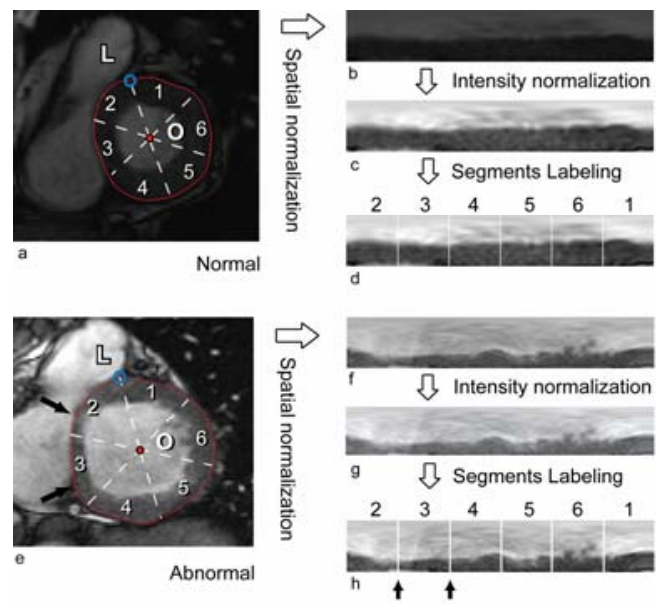

Fig. 3. Examples of the normalization procedure for a normal subject (a-d) and an abnormal wall motion patient (e-h). In a and e, the darker circular band is the myocardium, and the whiter pool is blood. In $\mathbf{a}$ and $\mathbf{e}, \mathrm{L}$ is the reference point and $\mathrm{O}$ is the centroid of the $\mathrm{LV}$. In $\mathbf{e}$ and $\mathbf{h}$, the two black arrows point to the abnormal segment.

Spatial Normalization. An essential feature of the LV is the approximately radial wall motion and circular epicardial boundary, therefore the analysis is simplified by mapping the pixel intensities from Cartesian ( $\mathrm{x}, \mathrm{y}$ ) to polar coordinates. In addition, the proposed mapping described below normalizes the size and shape of the myocardium by normalizing the length of each radial line from the LV centre to the epicardium. 
This normalizes the images spatially for both intra- and inter-subject comparisons. For slice $_{s t}$ of each subject, there are five steps:

Step 0. Set $c o l=1, \theta=\angle X O_{s t} L_{s t}, Q_{s t}=L_{s t} . c o l$ is defined as the output image column index.

Step 1. Plot line segment $O_{s t} Q_{s t}$ from $O_{s t}$ to $Q_{s t}$.

Step 2. Interpolate the pixel intensity values along line segment $O_{s t} Q_{s t}$ to $P$ points. Put the result in the $\mathrm{col}^{\text {th }}$ column of output image with $P$ rows.

Step 3. Set $\theta=\theta+\Delta \theta$ along the counterclockwise direction. Update $Q_{s t}$ (on the LV epicardium boundary) by constraint $\angle X O_{s t} Q_{s t}=\theta$. Set $\mathrm{col}=\mathrm{col}+1$.

Step 4. Repeat step1 and step3, until $Q_{s t}$ equals $L_{s t}$ (initial point).

After these steps, slice $_{s t}$ has been normalized to a $P \times c o l$ rectangular image. See Fig. $3 \mathrm{~b}$ and $3 \mathrm{f}$ for examples, with $\Delta \theta=1^{\circ}$, and $P=60$. The result is a $60 \times 360$ image. By means of this spatial normalization, LVs of different subjects were normalized to the same position, since for all of the resultant images the left edge represents the line from the LV centre to the reference point L, the row represents the radial distance (normalized by the distance to the epicardium for that radial line) and the column is the counterclockwise polar angle.

Intensity Normalization. In order to normalize the intensity differences across slices, phases, subjects and scanners, each pixel has its intensity set to $(x-\mu) / \sigma$, where, $x$ is the original intensity value, $\mu$ and $\sigma$ are respectively the mean and the standard deviation of the rectangular image. (See Fig.3c and 3g.)

Segments Labeling. According to the AHA segment model (Fig.1), the basal segments 1 to 6 correspond to the blocks found by dividing evenly the rectangular image along the vertical direction, as labeled in Fig. $3 \mathrm{~d}$ and $3 \mathrm{~h}$. The mid-cavity segments 7 to 12 have the same partitions as segments 1 to 6 . The apical segments 13 to 16 correspond to the $1^{\text {st }}-4^{\text {th }}$ rectangular block by dividing evenly the rectangular image along the vertical direction (Refer to [5]).

\section{Intra-segment Correlation Based Classification}

In the cardiac cycle, there are different phases alternating in a natural order, for instance, from systole to diastole. Motion patterns of the normal segment should be deviate away from motion patterns of the abnormal segment. For a specific segment, if we concatenate the normalized segment images of all the phases in column manner, we get a spatial-temporal segment image (e.g. Fig.4a, c). Comparing the motion pattern of the segment for normal (Fig. 4a) and abnormal cases (Fig. 4c), we found that from phase 4 to phase 18 , the normal segment contracts more than the abnormal segment. For the normalized segment image of each phase $t(t=1,2, \cdots, T)$, a column vector $U_{t}$ is calculated by averaging the vertical line intensity profile across $\theta$ angles of the segment (refer to Fig.2b). Subsequently the correlation coefficients $C C_{t}$ between $U_{E D}$ and 
$U_{t}(t=1,2, \cdots, T)$ can be calculated to measure the motion pattern along all the $t$ phases. ( $E D$ refers to the end diastolic phase, with $E D=1$ in Fig.4.) Fig. 4 d shows the correlation coefficients of both normal and abnormal segments. This demonstrates that the contraction is greater (correlation is lower) in a normal segment than an abnormal segment from phase 4 to 18 . Therefore, the $C C_{t}$ of the abnormal segment has a motion pattern that deviates from the normal segment's $C C_{t}(t=1,2, \cdots, T)$. Accordingly, the $C C_{t}$ measure can be use to discriminate the normal and abnormal segments, with a classification criterion derived from the training data. When a new patient is encountered, for each segment, the following steps summarized the recognition process: 1 . LV normalization; 2. Calculate $U_{t}(t=1,2, \cdots, T) ; 3$. Calculate $C C_{t}(t=1,2, \cdots, T) ; 4$. Classify to normal or abnormal based on a predefined classification criterion.
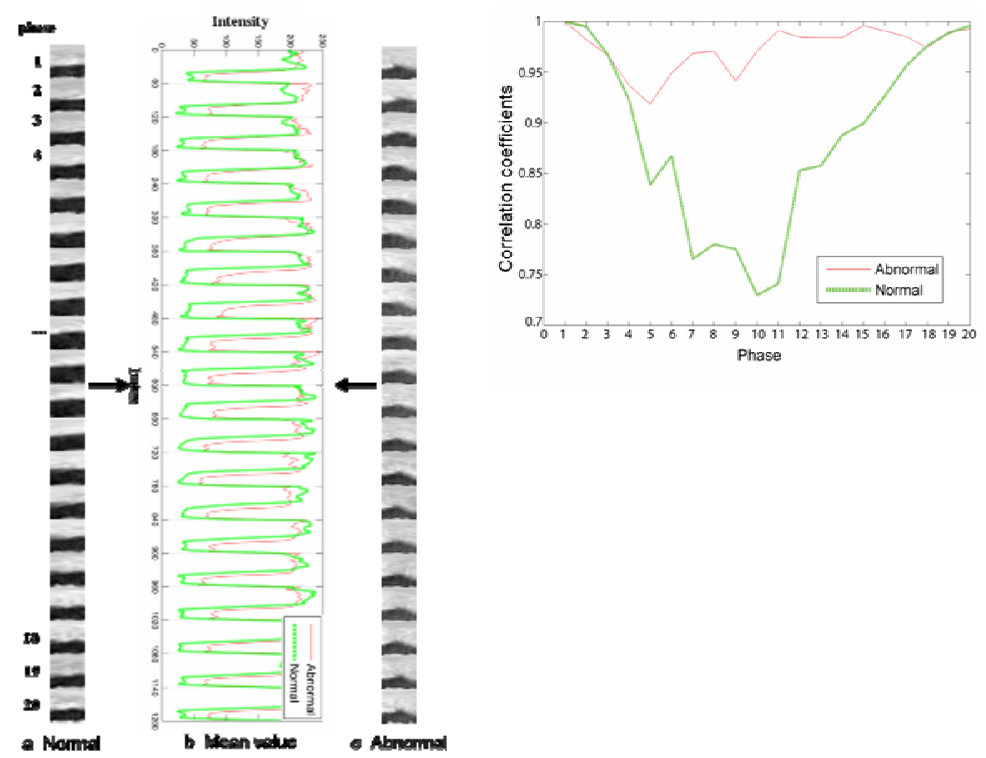

Fig. 4. Segment images from different phases concatenated in column from normal a) and abnormal c) subjects. b) $U$, intensity profiles of column image averaged across segment $\theta$ of the normal (green) and abnormal (red) segment. d) Intra-segment correlation coefficients of the normal and abnormal segment.

\section{Experiments and Results}

\subsection{Data}

For this study, the MRI of 17 patients were analyzed (2 female, 15 male, age: 64.8 \pm 9.5 ). Cine Fiesta MR short axis (SAX) images were obtained with a 1.5T GE Signa MRI. All the images were obtained during 10-15 s breath-holds with a temporal resolution of 20 cardiac phases. Six to $12 \mathrm{SAX}$ images were obtained from the atrioventricular ring to the apex (thickness $=8 \mathrm{~mm}$, gap $=8 \mathrm{~mm}, \mathrm{FOV}=320 \mathrm{~mm} \times 320 \mathrm{~mm}$, 
matrix $=256 \times 256$ ). For all subjects, a single basal slice was selected for this preliminary study, and 12/17 patients had regional abnormal wall motion for the basal slice by expert visual assessment. Before normalization, contour points were placed manually with spline interpolation to plot the epicardial boundary. From the epicardial boundary the centroid of the LV is calculated. For each subject, in the LV segmentation step, the first input landmark point at the ED phase is recorded as reference point $L$. In this work, the anterior end of the interventricular septum was used as the reference point. In the spatial normalization step $\Delta \theta$ was set to $1^{\circ}$ and $P$ was set to 60 , resulting in a $60 \times 360$ rectangular image in polar coordinates.

\subsection{Experiments and Results}

In order to determine a classification criterion for each segment, we calculated the $C C_{t}(t=1,2, \cdots, 20)$ averaged across all subjects with the same assessment (normal or abnormal wall motion). Fig 5 shows plots of the averaged $C C_{t}(t=1,2, \cdots, 20)$ for individual segments, demonstrating that the minimum value of the averaged normal $C C_{t}$ falls between phase 6 and 10 and is always smaller than the minimum of the averaged abnormal $C C_{t}$. Accordingly, for segment 1-6, the classification criterion is defined as: If $\min \left(C C_{t}\right)(t=6,7, \cdots, 10)<T_{\text {segment }}$ (segment $\left.=1,2, \cdots, 6\right)$, segment is classified as normal. Otherwise, the segment is classified as abnormal.
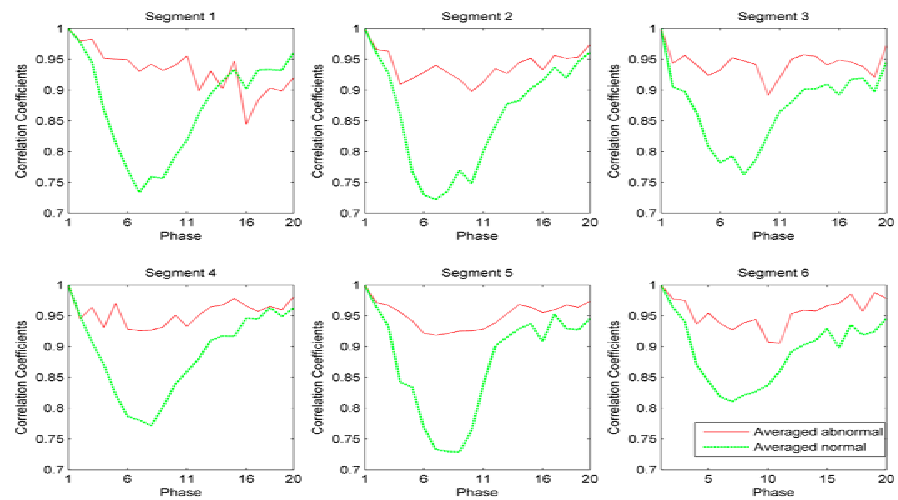

Fig. 5. Correlation coefficients. Each graph illustrates the wall motion of a segment as measured by the correlation $C C_{t}$ between the phase $t$ and end diastolic phase.

Table 1. Sensitivity, specificity and accuracy of segments 1-6

\begin{tabular}{lcccccc}
\hline & \multicolumn{7}{c}{ Segment } \\
\cline { 2 - 7 } & 1 & 2 & 3 & 4 & 5 & 6 \\
\hline Sensitivity & $80 \%$ & $85.7 \%$ & $100 \%$ & $75.0 \%$ & $85.7 \%$ & $100 \%$ \\
Specificity & $91.7 \%$ & $100 \%$ & $90.9 \%$ & $84.6 \%$ & $90 \%$ & $66.7 \%$ \\
Accuracy & $88.2 \%$ & $94.1 \%$ & $94.1 \%$ & $82.4 \%$ & $88.2 \%$ & $70.6 \%$ \\
Recognition time $\left(10^{-4} \mathrm{~s}\right)$ & 1.4 & 1.7 & 1.7 & 1.1 & 1.1 & 1.8 \\
\hline
\end{tabular}


Table 2. Comparison with manual and other computer aided method

\begin{tabular}{lccc}
\hline & Manual method in [1] & computer aided Method in [1] & Proposed method \\
\hline Mean Sensitivity & $80 \%$ & $84 \%$ & $87.1 \%(27 / 31)$ \\
Mean Specificity & $76 \%$ & $77 \%$ & $85.9 \%(61 / 71)$ \\
Mean Accuracy & $77 \%$ & $79 \%$ & $86.3 \%(88 / 102)$ \\
\hline
\end{tabular}

The performance of the proposed classification method was calculated on a per segment basis, where the expert reader's visual assessment served as ground truth. Segment classification results were used to calculate the sensitivity, specificity and accuracy, with a total of $17 \times 6=102$ segments (Table 1 ). Table 1 also shows that the computational time for recognition (normalization and discrimination) is approximately $10^{-4}$ second per segment on consumer hardware $(2 \times 2.8 \mathrm{GHz}$ Quad-core Intel Xeon Mac Pro, Apple) with non-optimized Matlab code (Mathworks). In Table 2, the performance of the proposed method was also evaluated by comparisons with previous work in [1]. These preliminary results are better than the manual and computer aided methods described in [1] as measured by sensitivity, specificity and accuracy.

\section{Discussions and Conclusions}

With the normalization scheme, LV images of different positions, sizes, shapes and intensity levels are normalized to myocardium coordinates with the same position, size, and intensity level, providing a foundation for pattern recognition of wall motion. Spatial correspondence is established for segments rather than pixels in this work. Although this does not provide for pixel-wise analysis of cardiac motion of non-rigid registration techniques [7], it is suitable for automated recognition of regional LV wall motion because the wall motion assessment required clinically is based on the AHA segment model [5,6]. This partially solves the second problem proposed in the introduction. In order to solve the third and fourth problems, for each segment, an intra-subject correlation measure was proposed. Since the correlation coefficients are calculated with an intra-segment manner, the variability of myocardial thickness between subjects and the papillary muscles, trabeculations' size and position will not be an issue.

Although the mean specificity and accuracy were relatively low for clinical use, as a preliminary attempt to use the pattern recognition method for assessment of regional LV wall motion, the results are still promising. Further improvements are desirable, including: 1) combining with automated or semi-automated methods to segment the LV;2) training with mid-cavity and apical slices where papillary muscles are present; 3) a larger set of subjects, with separate training and validation sets; 4) more efficient methods of classification (e.g. support vector machine [8]). While a simple classification criterion was used in this work, the results are still promising due to the effectiveness of the normalization and feature extraction scheme.

Compared with the previous work on regional LV wall motion analysis methods $[1,2,3,4]$, main characteristics of the proposed method can be summarized as: 1) Segmentation of the endocardium is not required. Analyzing the radial profile in LV coordinates has been shown to be sufficient for detecting wall motion abnormalities in this patient study data set. 2) This method finds an appropriate balance between 
complexity and simplicity. A pixel-wise estimate of wall motion adds complexity of analysis and the detailed motion is not required for clinical assessment. An accurate location of the reference point to demarcate segments of each phase is difficult. Therefore, the proposed reference point location scheme was utilized, although it will lead to slight segment location errors due to the LV twisting and ventricular torsion. 3) The complete information from all phases and segments is utilized to determine a wall motion estimate comparable to the standard manual wall motion scores. 4) This method is a novel application of pattern recognition techniques to LV motion analysis.

In summary, a scheme has been proposed for abnormal LV wall motion detection by the techniques of pattern recognition. By the proposed LV normalization method, the LVs with different shapes, positions, sizes and intensity levels are normalized to rectangular images with same position, size and intensity level. This normalization scheme is a crucial bridge for the further applications of pattern recognition method to LV motion analysis. Following the normalization, an intra-segment correlation based classifier was used for recognition of segments with abnormal regional LV wall motion. The results demonstrate a promising method for recognition of abnormal LV wall motion that deserves more extensive validation.

\section{References}

1. Caiani, E.G., Toledo, E., MacEneaney, P., et al.: Automated interpretation of regional left ventricular wall motion from cardiac magnetic resonance images. Journal of Cardiovascular Magnetic Resonance 8(3), 427-433 (2006)

2. Saring, D., Ehrhardt, J., Stork, A., et al.: Computer-assisted analysis of 4 D cardiac MR image sequences after myocardial infarction. Methods Inf. Med. 45(4), 377-383 (2006)

3. Suinesiaputra, A., Uzumcu, M., Frangi, A.F.: Detecting regional abnormal cardiac contraction in short-axis MR images using independent component analysis. In: Barillot, C., Haynor, D.R., Hellier, P. (eds.) MICCAI 2004. LNCS, vol. 3216, pp. 737-744. Springer, Heidelberg (2004)

4. Suinesiaputra, A., Frangi, A.F., Uzumcu, M.: Extraction of myocardial contractility patterns from short-axes MR images using independent component analysis. In: Sonka, M., Kakadiaris, I.A., Kybic, J. (eds.) CVAMIA/MMBIA 2004. LNCS, vol. 3117, pp. 75-86. Springer, Heidelberg (2004)

5. Cerqueira, M.D., Weissman, N.J., Dilsizian, V.: Standardized myocardial segmentation and nomenclature for tomographic imaging of the heart. J. Nucl. Cardiol. 9, 240-245 (2002)

6. Bogaert, J., Dymarkowski, S., Taylor, A.M.: Clinical Cardiac MRI, pp. 95-97. Springer, Heidelberg (2005)

7. Li, B., Young, A.A., Cowan, B.R.: GPU accelerated non-rigid registration for the evaluation of cardiac function. Med. Image Comp. Comp. Assist. Interv. 11(Pt 2), 880-887 (2008)

8. Burges, C.J.C.: A Tutorial on Support Vector Machines for Pattern Recognition. Data Mining and Knowledge Discovery 2, 121-167 (1998)

9. Fradkin, M., Ciofolo, C., Mory, B., Hautvast, G., Breeuwer, M.: Comprehensive segmentation of cine cardiac MR images. Med. Image Comp. Comp. Assist. Interv. 11(Pt 1), 178185 (2008)

10. Ben Ayed, I., Lu, Y., Li, S., Ross, I.: Left ventricle tracking using overlap priors. Med. Image Comp. Comp. Assist. Interv. 11(Pt 1), 1025-1033 (2008) 
11. Cocosco, C.A., Niessen, W.J., Netsch, T., Vonken, E.J., Lund, G., Stork, A., Viergever, M.A.: Automatic image-driven segmentation of the ventricles in cardiac cine MRI. J. Magn. Reson. Imaging 28(2), 366-374 (2008)

12. Lynch, M., Ghita, O., Whelan, P.F.: Segmentation of the Left Ventricle of the Heart in 3D+t MRI Data Using an Optimized Nonrigid Temporal Model. IEEE Trans. Med. Imaging 27(2), 195-203 (2008)

13. Lu, Y., Radau, P., Connelly, K., Dick, A., Wright, G.M.: Segmentation of Left Ventricle in Cardiac Cine MRI: An Automatic Image-Driven Method. LNCS, vol. 5528, pp. 339-347. Springer, Heidelberg (2009) 